\title{
Estudio comparativo entre tobramicina y tobramicina más ciprofloxacino como profilaxis para la biopsia transrectal de próstata
}

\author{
Bosquet Sanz M, Gimeno Argente V, Arlandis Guzmán S, Bonillo García MÁ, Trassierra Villa M, \\ Jiménez Cruz JF.
}

Servicio de Urología. Hospital Universitario La Fe. Valencia.

Actas Urol Esp. 2006:30(9):866-870

\section{RESUMEN}

\section{ESTUDIO COMPARATIVO ENTRE TOBRAMICINA Y TOBRAMICINA MÁS CIPROFLOXACINO COMO PROFILAXIS PARA LA BIOPSIA TRANSRECTAL DE PRÓSTATA}

Introducción y Objetivos: Estudio de eficacia en la prevención de complicaciones infecciosas tras biopsia transrectal de próstata. Revisión de las complicaciones infecciosas y microorganismo implicados.

Material y métodos: Estudio prospectivo y aleatorizado en 153 pacientes en los que se realizaron 157 biopsias de próstata. El Grupo A (71 pacientes) recibieron tobramicina $100 \mathrm{mg}$ una dosis intravenosa media hora antes de la biopsia y otra intramuscular a las 8 horas de la primera, y los del Grupo B (85 pacientes) recibieron el mismo esquema de tobramicina y añadiendo ciprofloxacino oral $500 \mathrm{mg}$ media hora antes de la biopsia continuando luego con una dosis cada 12 horas durante tres días.

Resultados: en el Grupo A se realizaron 71 biopsias y en el Grupo B 86. 50 pacientes (31,8\%) presentaron hematuria, $20(12,7 \%)$ fiebre, $15(9,5 \%)$ hemospermia, $7(4,4 \%)$ dolor perineal, uno $(1,2 \%)$ orquiepididimitis y otro $(1,2 \%)$ retención urinaria. De los pacientes con fiebre 15 pertenecen al grupo A y 5 al grupo B (p=0,004). Ingresaron por fiebre $15(21,1 \%)$ pacientes del grupo A y $3(3,5 \%)$ del grupo B (p=0,0006). En el 67\% de los hemocultivos se aisló $E$. Coli.

Conclusiones: La pauta profiláctica consistente en tobramicina más ciprofloxacino resultó ser más eficaz en la prevención de ingresos por fiebre post-biopsia que la tobramicina sola. La complicación más frecuente fue la hematuria. El microorganismo más frecuentemente fue E. Coli sensible, en nuestro hospital, a amoxicilina-clavulánico, tobramicina y cefalosporinas de tercera generación.

Palabras clave: Biopsia transrectal ecodirigida de próstata.

\section{ABSTRACT \\ COMPARATIVE STUDY BETWEEN TOBRAMICIN AND TOBRAMICIN PLUS CIPROFLOXACIN IN TRANSRECTAL PROSTATE BIOPSY PROPHYLAXIS}

Introduction and objectives: To compare the efficacy of tobramicin and tobramicin + ciprofloxacin for prevention of transrectal prostatic biopsy infectious complications. We revised our complications, microorganism most common in the infectious complications, and their sensibility.

Material and methods: Prospective and randomized study in 153 patients with 157 prostatic biopsies. The 71 patients in group A were treated with intramuscular tobramicin $100 \mathrm{mg}$, one dose 30 minutes before biopsy and another one 8 hours afterwards. The 85 patients in group B were treated with the same tobramicin doses and oral ciprofloxacin $500 \mathrm{mg}$, one dose 30 minutes before biopsy and afterwards they continue with the ciprofloxacin every 12 hours during 3 days.

Results: we did 71 biopsies in group A and 86 in group B. $50(31,8 \%)$ patients had hematuria, $20(12,7 \%)$ fever, $15(9,5 \%)$ hemospermia, $7(4,4 \%)$ perineal pain, one $(1,2 \%)$ orchiepididymitis and another one $(1,2 \%)$ urinary retention. The patients who had fever were 15 of the group A and 5 of the group B (p=0,004). A total of $15(21,1 \%)$ patients with fever of the group A needed to be treated in the hospital and 3 patients $(3,5 \%)$ of the group B $(\mathrm{p}=0,0006)$. E. coli growthed in $67 \%$ of the blood cultures and amoxicillin-clavulanic, tobramicin and third generation of cephalosporins were the antibiotics more eficacious.

Conclusions: Prophylaxis scheme with tobramicin plus ciprofloxacin was more efficacy that tobramicina alone in transrectal prostatic biopsy. Hematuria was the most common complication. E.coli was the microorganism most frequent in infectious complications after prostatic biopsy and amoxicillin-clavulanic, tobramicin and third generation of cephalosporins the most effective antibiotics in our hospital.

Keywords: Ultrasound guided transrectal prostatic biopsy. 
L a biopsia transrectal ecodirigida de próstata es un procedimiento bien tolerado y seguro, aunque no exento de complicaciones, entre las que destacan hematuria, hemospermia, rectorragia e infecciones $^{1}$. No obstante, pese a que las complicaciones infecciosas post-biopsia son bien conocidas y potencialmente fatales en algunas ocasiones ${ }^{2}$, no existe consenso en la profilaxis que ha de ser utilizada.

La mayoría de trabajos apuntan la necesidad de realizar profilaxis antibiótica al efectuar una biopsia transrectal de próstata, aunque la gran diversidad de pautas existente en la literatura hace que la vía de administración, el antibiótico y la duración de la pauta profiláctica ideal esté todavía por determinar. Probablemente esta diversidad en los esquemas profilácticos sea debido a que, aun siendo el patógeno más frecuentemente implicado el mismo, el E. Coli ${ }^{3}$, la sensibilidad a los antibióticos no es la misma en función del ámbito geográfico o incluso hospitalario.

Debido al aumento de los ingresos por sepsis post-biopsia prostática observado en nuestro servicio, decidimos realizar un estudio prospectivo, aleatorizado y de grupos paralelos, para comparar la eficacia de tobramicina sola frente a tobramicina más ciprofloxacino en la prevención de complicaciones infecciosas derivadas de la biopsia prostática transrectal.

\section{MATERIAL Y MÉTODOS}

Durante el periodo comprendido entre mayo y septiembre de 2004, se realizaron un total de 157 biopsias de próstata a 153 pacientes (a cuatro se les repitió la biopsia). La indicación de biopsia fue elevación de PSA (>4 ng/ml) y/o tacto rectal sospechoso.

Los pacientes se distribuyeron en dos grupos de forma aleatoria: en el Grupo A se realizaron 71 biopsias (45,2\%), utilizando tobramicina $100 \mathrm{mg}$ una dosis intravenosa media hora antes de la biopsia y otra intramuscular a las 8 horas de la primera; en el Grupo B se incluyeron 86 (54,8\%), empleando el mismo esquema de tobramicina y añadiendo ciprofloxacino oral $500 \mathrm{mg}$ media hora antes de la biopsia continuando luego con una dosis cada 12 horas durante tres días.

Se efectuaron biopsias sextantes aleatorias, tomando muestras de la zona periférica craneal, medial y caudal de ambos lóbulos, y en aquellos casos en los que en la ecografía se visualizaba una zona sospechosa se tomaron una o dos biopsias selectivas adicionales. Todas las biopsias fueron ecodirigidas con un ecógrafo Siemens ${ }^{\circledR}$ Sonoline
Antares, con transductor endorectal EC9-4 multifrecuencia (3,9 y $8 \mathrm{Mhz})$. Utilizamos pistolas de biopsia automáticas de $18 \mathrm{G}$ de calibre, que obtienen cilindros de $2 \mathrm{~cm}$ de longitud.

En aquellos pacientes que tomaban antiagregantes, éstos fueron suspendidos siete días antes de la biopsia, y los que tomaban anticoagulantes se reajustó la pauta por parte del Servicio de Hematología. Se efectuó preparación intestinal previa administrando un enema de limpieza la noche antes y la misma mañana de la biopsia, que se realizó de forma ambulatoria, a través de la Unidad de Cirugía sin Ingreso (UCSI). A los pacientes se les canalizó una vía periférica para la administración intravenosa de tobramicina. Como pauta analgésica se utilizaron $100 \mathrm{mg}$ de petidina iv. Una vez realizada la biopsia y comprobada la ausencia de complicaciones inmediatas significativas se procedía al alta del paciente.

Se recogieron en una base de datos y de forma prospectiva los datos administrativos del paciente, patología previa, pauta antibiótica administrada, complicaciones inmediatas (reacción vasovagal, uretrorragia, rectorragia, etc.) y tardías (hematuria, hemospermia, rectorragia, fiebre, shock séptico, dolor perineal, orquiepididimitis, retención urinaria, etc.).

Se registraron los ingresos por sepsis de origen urinario en cada grupo, considerándolos como variable de eficacia clínica principal. A los pacientes que ingresaron por sepsis de origen urinario, se les extrajeron muestras de sangre y orina para bacteriología, y entre los cultivos que fueron positivos se identificó el microorganismo aislado y su antibiograma.

Las diferencias porcentuales se analizaron mediante el test Chi-cuadrado, utilizando como nivel de significación estadística una $\mathrm{p}<0,05$.

\section{RESULTADOS}

En el grupo A se realizaron un total de 71 biopsias a 71 pacientes diferentes, en el grupo B se realizaron 86 biopsias a 85 pacientes (a uno se le repitió la biopsia). A tres pacientes se les repitió la biopsia, siendo incluidos en pautas diferentes.

La edad media de los pacientes fue $65 \pm 7$ años, sin observarse diferencias significativas entre los dos grupos terapéuticos.

Entre las complicaciones inmediatas a la biopsia tan sólo destacar un paciente del grupo B que presentó un cuadro vasovagal con hipotensión que se resolvió administrando fluidos y atropina intravenosa. 
Respecto a las complicaciones Tabla 1

tardías la más frecuente fue la hematuria, que no requirió ingreso ni necesidad de transfusión en ninguno de los pacientes, con cincuenta casos $(31,8 \%)$. Apareció fiebre en veinte pacientes $(12,7 \%) \mathrm{y}$ hemospermia en quince $(9,5 \%)$. Siete pacientes $(4,4 \%)$ refirieron dolor o molestias perineales, uno orquiepididimitis y otro paciente retención aguda de orina (RAO) que precisó sondaje (Tabla 1). 85 pacientes no presentaron ninguna complicación (54, 1\%).

Complicaciones post-biopsia en función de grupo profiláctico.

\begin{tabular}{lccccc}
\hline Complicación & \multicolumn{2}{c}{ Grupo A } & \multicolumn{2}{c}{ Grupo B } & \multicolumn{2}{c}{ Valor de } \\
& No Biop & \% & No Biop & \% & p \\
\hline Hematuria & 24 & 33,8 & 26 & 30,2 & 0,63 \\
Fiebre & 15 & 21,1 & 5 & 5,8 & 0,004 \\
Hemospermia & 8 & 11,3 & 7 & 8,1 & 0,507 \\
Dolor perineal & 4 & 5,6 & 3 & 3,5 & 0,517 \\
Orquiepididimitis & 0 & 0 & 1 & 1,2 & - \\
RAO & 0 & 0 & 1 & 1,2 & - \\
\hline
\end{tabular}

№ Biop: Número de biopsias; \%: porcentaje de biopsias respecto al total del grupo; p: Significación estadística con el test Chi-cuadrado

De los veinte pacientes que pre- Grupo A: Tobramicina, Grupo B: Tobramicina + Ciprofloxacino sentaron fiebre, dos consultaron al médico de cabecera y se pautó tratamiento antibiótico ambulatorio. El resto acudió a urgencias de nuestro hospital, procediendo a su ingreso (Tabla 2) para tratamiento antibiótico intravenoso empírico y a todos se les extrajeron muestras para cultivo de sangre y orina. Ninguno de ellos precisó ingreso en UCI.

Respecto al resultado de los urinocultivos nos encontramos con un alto porcentaje (45\%) de éstos contaminados, de tal forma que tan sólo cuatro de los dieciocho urinocultivos fueron positivos, siendo el microorganismo aislado en todos ellos E. coli. En relación a los hemocultivos fueron positivos el 67\%, aislando en diez de los doce hemocultivos positivos el mismo microorganismo E. coli. (Fig. 1).

Respecto al antibiograma del microorganismo aislado con más frecuencia, E. coli, los antibióticos que más sensibilidad, y por ende menos resistencia, tienen al mismo son Amoxicilina-clavulánico, Tobramicina y Cefotaxima/Ceftriaxona. El Ciprofloxacino presentó una resistencia a E. coli del $40 \%$ (Fig. 2).

De los dieciocho pacientes que ingresaron por fiebre quince pertenecían al grupo A (tobramicina sola) y tres al grupo B (tobramicina más ciprofloxacino). Estos resultados fueron estadísticamente significativos $(\mathrm{p}=0,0006)$.

\section{DISCUSIÓN}

El cáncer de próstata constituye el cáncer más frecuente en el varón y la segunda causa de mortalidad oncológica por detrás del cáncer de pulmón ${ }^{4}$. De ahí la gran importancia que adquieren los programas de detección precoz en este tipo de cáncer, en los que desempeñan un papel crucial el PSA y la biopsia de próstata. De entre los métodos para la

Tabla 2

Ingresos por fiebre de origen urinario.

\begin{tabular}{lccc}
\hline & $\begin{array}{c}\text { Pauta A } \\
\text { n (\%) }\end{array}$ & $\begin{array}{c}\text { Pauta B } \\
\text { n (\%) }\end{array}$ & $\begin{array}{c}\text { Total } \\
\text { n (\%) }\end{array}$ \\
\hline No & $56(78,9)$ & $83(96,5)$ & $139(88,5)$ \\
Sí & $15(21,1)$ & $3(3,5)$ & $18(11,5)$ \\
Total & $71(45,2)$ & $86(54,8)$ & $157(100)$ \\
\hline
\end{tabular}

Número absoluto de los pacientes y entre paréntesis el porcentaje respecto al total del grupo

Pauta A: Tobramicina, Pauta B: Tobramicina + Ciprofloxacino

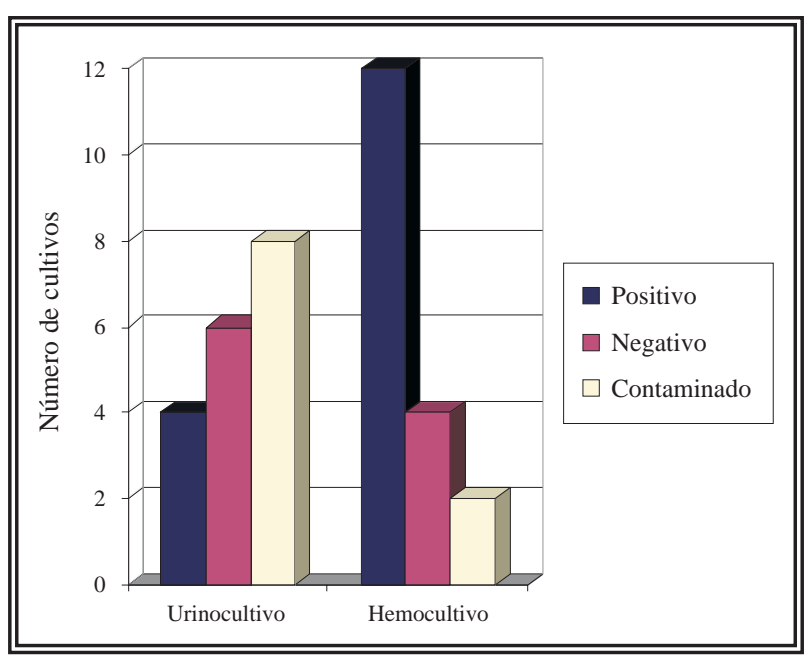

FIGURA 1. Urinocultivos y Hemocultivos

realización de una biopsia prostática, la biopsia transrectal ecodirigida es el más extendido hoy en día y, como hemos observado en este estudio, es una técnica no exenta de complicaciones, pues cerca de la mitad de nuestros pacientes presentaron algún tipo de complicación. De hecho, algunos auto- 


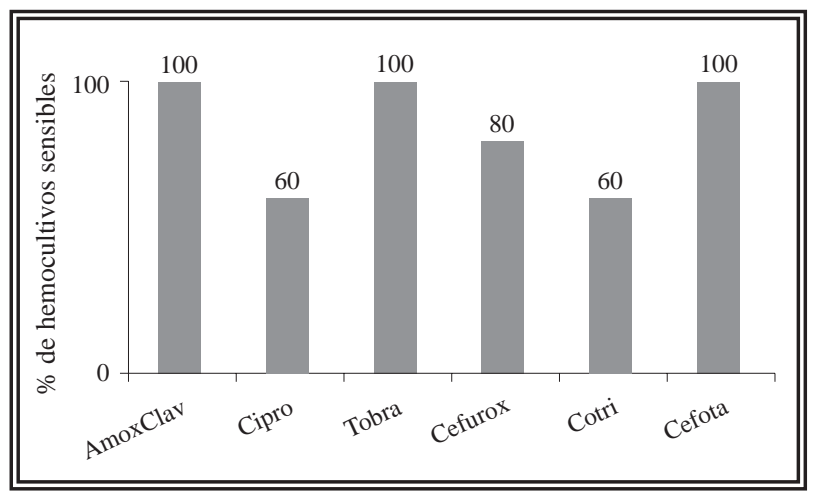

FIGURA 2. Sensibilidad de E. coli.

AmoxClav: Amoxicilina-Clavulánico.; Cipro: Ciprofloxacino;

Tobra: Tobramicina; Cefurox: Cefuroxima; Cotri: Cotrimoxazol;

Cefota: Cefotaxima/Ceftriaxona.

res, debido a la frecuencia de este tipo de complicaciones, han llevado a considerarlas más que complicaciones efectos intrínsecos de la propia técnica ${ }^{5}$. La mayoría de estas complicaciones suelen ser menores tales como hematuria, hemospermia, rectorragia, molestias perineales, o incluso cuadros de retención urinaria que precisan sondaje vesical ${ }^{1}$.

En nuestra serie tuvimos una cifra de complicaciones no infecciosas similar a la ofrecida por otros autores (Tabla 3), si bien encontramos una menor tasa de hemospermia, quizás porque en nuestro servicio no efectuamos biopsia de vesículas seminales de forma sistemática.

Respecto a las complicaciones infecciosas, éstas tienen una incidencia muy dispar de unas series a otras, probablemente en relación al antibiótico utilizado como profilaxis y a la resistencia tan dependiente del área geográfica que tiene $E$. coli. Las series más recientes consultadas reflejan una incidencia entre $0,67 \%$ y 9,6\%1,6,9,10. La mayoría de estas complicaciones infecciosas suelen ser menores y bien toleradas por el paciente, consisten en fiebre provocada por una bacteriemia transitoria, aunque en algunas situaciones pueden llegar a tener consecuencias importantes e incluso comprometer la vida del paciente, pues pueden dar lugar a la aparición de cuadros sépticos graves que requieren el ingreso del paciente en $\mathrm{UCI}^{1}$, y abscesos que obliguen a tratamiento quirúrgico urgente ${ }^{2}$.

Si bien las complicaciones no infecciosas de nuestro estudio son similares a las de las series consultadas, no ocurre lo mismo con las complicaciones infecciosas, pues hemos tenido una tasa global de fiebre post-biopsia del $12,7 \%$, notablemente superior al resto de series consultadas (Tabla 3). La mayoría de casos de fiebre post-biopsia que precisó ingreso hospitalario se produjo en el grupo A (tobramicina sola como pauta profiláctica) (Tabla 2). En el grupo B, en el que utilizamos la tobramicina asociada a ciprofloxacino, hemos tenido una tasa de ingresos por fiebre más acorde con los resultados publicados en la literatura. Estas diferencias resultaron estadísticamente significativas, lo que explicaría que la pauta que estábamos utilizando había perdido eficacia para la prevención de este tipo de complicaciones, o simplemente la tobramicina + ciprofloxacino es una pauta mejor ya que al ser dos antibióticos asociados los microorganismos que son resistentes a uno son cubiertos por el otro. Sin embargo la alta tasa de resistencia de $E$. coli, en nuestro medio, a ciprofloxacino, hace dificil entender la mayor eficacia de la asociación. Especulativamente podríamos considerar tres apreciaciones: 1. Sinergismo entre ambos antimicrobianos, lo que potencia la acción de ambos, 2. La alta dosis de ciprofloxacino $500 \mathrm{mg}$ obtiene concentraciones muy altas en orina, que podría superar la concentración mínima inhibitoria (CMI) establecida in vitro, 3. Las dos dosis de tobramicina no cubren el riesgo en el tiempo, produciéndose la infección después de 24 horas de la administración. Estos resultados nos obligaron a adoptar la asociación ciprofloxacino más tobramicina como pauta profiláctica habitual en la realización de la biopsia transrectal de próstata en nuestro servicio.

Tabla 3

Porcentaje de complicaciones en diferentes series publicadas.

\begin{tabular}{lccccc}
\hline Referencia & Hematuria & Hemospermia & Fiebre & RAO & Rectorragia \\
\hline Santos Arrontes et al. $^{1}$ & 52,9 & 40,6 & 6,64 & 1,66 & 33,5 \\
Rietbergen et al. $^{6}$ & 23,6 & 45,3 & 4,2 & - & - \\
Enlund et al. $^{7}$ & 49,6 & - & 2,9 & 0,24 & 21,7 \\
Raaijmakers et al. $^{8}$ & 22,6 & 50,4 & 3,5 & 0,4 & - \\
Hospital La Fe & 31,8 & 9,5 & 12,7 & 0,64 & - \\
\hline
\end{tabular}


A pesar de que el porcentaje de urinocultivos contaminados es alto, probablemente debido a la mala recogida de las muestras de orina en las áreas de observación de urgencias, y que hemos obtenido pocos hemocultivos positivos para hacer un estudio concluyente, sí que encontramos que el microorganismo aislado y responsable con más frecuencia de este tipo de complicaciones fue, como así coincide en otras series, Escherichia coli ${ }^{3}$.

Sin pretender realizar un estudio microbiológico exhaustivo, pues el objetivo principal del presente estudio fue clínico y la muestra de la que disponemos para su análisis no es lo suficientemente grande para ello ya que tan sólo diez hemocultivos fueron positivos a E. coli, observamos que en nuestro Hospital, entre los antibióticos testados, los que más sensibilidad ofrecen al mismo son amoxicilinaclavulánico, tobramicina y las cefalosporinas de tercera generación como cefotaxima o ceftriaxona (Fig. 2). El ciprofloxacino presenta, según nuestros resultados, una tasa de resistencia del $40 \%$, por lo que convendría plantear en el futuro un análisis comparativo entre tobramicina y amoxicilina-clavulánico o alguna de las cefalosporinas de tercera generación, o bien establecer una profilaxis con tobramicina de mayor duración.

\section{CONCLUSIONES}

1. En nuestro medio la biopsia transrectal de próstata es una técnica que presenta un índice de complicaciones importante (45,9\%), aunque en su mayoría son leves.

2. La complicación más frecuente es la hematuria $(31,8 \%)$.

3. El microorganismo más frecuentemente implicado en las complicaciones infecciosas post-biopsia es Escherichia coli. Los antibióticos más eficaces al mismo son, en nuestro hospital, amoxicilina-clavulánico, tobramicina y cefalosporinas de tercera generación.

4. La pauta profiláctica consistente en tobramicina más ciprofloxacino resultó ser más eficaz en la prevención de ingresos por fiebre post-biopsia que la tobramicina sola.

\section{REFERENCIAS}

1. Santos Arrontes D, Luján Galán M, Pascual Mateo C, Chiva Robles V, Fernández González I, Berenguer Sánchez A. Análisis descriptivo de los efectos adversos asociados a la biopsia transrectal de próstata después de 603 procedimientos. Arch Esp Urol. 2004;57(6):601-605.

2. Borer A, Gilad J, Sikuler E, Riesenberg K, Schlaeffer F, Buskila D. Fatal Clostridium sordellii ischiorectal abscess with septicaemia complicating ultrasoundguided transrectal prostate biopsy. J Infect. 1999;38(2):128-129.

3. Tal R, Livne PM, Lask DM, Daniel J. Empirical management of urinary tract infections complicating transrectal ultrasound guided prostate biopsy. J Urol. 2003;169(5): 1762-1765.

4. www.ine.es.

5. Herranz Amo F, Rodriguez Fernandez E, Diez Cordero JM, Lledo Garcia E, Verdu Tartajo F, Gonzalez Chamorro F, Subira Castillon C. Morbidity of and tolerance to ultrasonography-guided transrectal biopsy of the prostate. Actas Urol Esp. 1996;20(10):858-866.

6. Rietbergen JB, Boeken Kruger AE, Kranse R, Schröeder FH. Complications of trasrectal ultrasound-guided systematic sextant biopsies of the prostate: evaluation of complication rates and risk factors within a population-based screening program. Urology. 1997;49(6):875-880.

7. Enlund AL, Varenhorst E. Morbidity of ultrasound-guided transrectal core biopsy of the prostate without prophylactic antibiotic therapy. A prospective study in 415 cases. $\mathrm{Br}$ J Urol. 1997;79(5):777-780.

8. Raaijmakers R, Kirkels WJ, Roobol MJ, Wildhagen MF, Schrder FH. Complication rates and risk factors of 5802 transrectal ultrasound-guided sextant biopsies of the prostate within a population-based screening program. Urology 2002;60(5):826-830.

9. Shandera KC, Thibault GP, Deshon GE. Efficacy of one dose fluorquinolone before prostate biopsy. Urology 1998;52(4):641-643.

10. Tobias-Machado M, Correa TD, De Barros EL, Wroclawski ER. Antibiotic prophylaxis in prostate biopsy. A comparative randomized clinical assay between ciprofloxacin, norfloxacin and chloramphenicol. Int Braz J Urol. 2003;29(4): 313-319.

Dr. M. Bosquet Sanz

Servicio de Urología.Hospital Universitario La Fe

Avda. Campanar no 21

46009 Valencia

e-mail: manubosquet@hotmail.com

(Trabajo recibido el 19 de julio de 2006) 